\title{
Review Article \\ Respiratory Gating for Radiotherapy: Main Technical Aspects and Clinical Benefits
}

\author{
Philippe Giraud ${ }^{1}$ and Annie Houle ${ }^{2}$ \\ ${ }^{1}$ Department of Radiation Oncology, Paris Descartes University, European Georges Pompidou Hospital, \\ 75015 Paris, France \\ ${ }^{2}$ Department of Radiation Oncology, Centre Intégré de Cancérologie de Laval (CICL), Hôpital de la Cité-De-La-Santé, \\ 1755 Boulevard René-Laennec, Laval, QC, Canada H7M 3L9
}

Correspondence should be addressed to Philippe Giraud; philippe.giraud@egp.aphp.fr

Received 8 January 2013; Accepted 29 January 2013

Academic Editors: A. S. Melani, C. Owen, and B. A. Rybicki

Copyright (C) 2013 P. Giraud and A. Houle. This is an open access article distributed under the Creative Commons Attribution License, which permits unrestricted use, distribution, and reproduction in any medium, provided the original work is properly cited.

\begin{abstract}
Respiratory-gated radiotherapy offers a significant potential for improvement in the irradiation of tumor sites affected by respiratory motion such as lung, breast, and liver tumors. An increased conformality of irradiation fields leading to decreased complication rates of organs at risk is expected. Five main strategies are used to reduce respiratory motion effects: integration of respiratory movements into treatment planning, forced shallow breathing with abdominal compression, breath-hold techniques, respiratory gating techniques, and tracking techniques. Measurements of respiratory movements can be performed either in a representative sample of the general population, or directly on the patient before irradiation. Reduction of breathing motion can be achieved by using either abdominal compression, breath-hold techniques, or respiratory gating techniques. Abdominal compression can be used to reduce diaphragmatic excursions. Breath-hold can be achieved with active techniques, in which airflow of the patient is temporarily blocked by a valve, or passive techniques, in which the patient voluntarily breath-holds. Respiratory gating techniques use external devices to predict the phase of the breathing cycle while the patient breathes freely. Another approach is tumor-tracking technique, which consists of a real-time localization of a constantly moving tumor. This work describes these different strategies and gives an overview of the literature.
\end{abstract}

\section{Introduction}

Taking into account the respiratory movements has always been a major concern of thoracic radiotherapy. The development of conformal radiotherapy using reduced radiation fields, with or without intensity modulation, and above all the growing interest for hypofractionated stereotactic body radiotherapy, further enhanced this concern [1-4]. In 1987, an American team noticed that treatment in deep inspiration spared parts of the lungs, and they suggested a need to develop "Radiotherapy Gated to Respiration" [5]. The term "gating" was subsequently used to designate a variety of different practices. The first technique designed to control respiratory movements was developed in Japan in 1989. It consisted of an airbag system designed to synchronize radiotherapy, but it was not further developed [6]. Five main strategies are currently used to reduce respiratory motion effects: integration of respiratory movements into treatment planning (geometrical or dosimetric), forced shallow breathing with abdominal compression, breath-hold techniques (active or voluntary), respiratory gating techniques, and tracking techniques.

Traditionally, according to International Commission on Radiation Units and Measurements (ICRU) [7, 8] recommendations, tumor motion is taken into account by adding a specific security margin (internal margin) around the clinical target volume (CTV), in order to create the internal target volume (ITV). Positioning uncertainties are then added to create the planning target volume (PTV). However, this strategy has its limits. For tumors with significant respiratory motion, such as those near the diaphragm, the addition of various geometric margins leads to irradiation of a large volume of healthy tissue, increasing the risk of complications, and therefore limiting the possibility of dose escalation (Table 1). 
TABLE 1: Main studies reporting movements of organs during free breathing in the literature.

\begin{tabular}{|c|c|c|c|}
\hline Authors & Localization & Free Breathing & Deep Breath-Hold \\
\hline $\begin{array}{l}\text { Suramo et al. [9], Davies et al. [10], Balter et al. [11], } \\
\text { Wagman et al. [12], Shimizu et al. [13] }\end{array}$ & Liver & $16.7 \mathrm{~mm}(5-40)$ & $35.0 \mathrm{~mm}(\mathrm{CC})(12-80)$ \\
\hline $\begin{array}{l}\text { Davies et al. [10], Balter et al. [11], Giraud et al. [14], } \\
\text { Ford et al. [15], Wagman et al. [12] }\end{array}$ & Diaphragms & $11.9 \mathrm{~mm}(7-38.2)$ & $39 \mathrm{~mm}(\mathrm{CC})(3.1-95)$ \\
\hline $\begin{array}{l}\text { Ohara et al. [6], Barnes et al. [16], Ekberg et al. [17], } \\
\text { Giraud et al. [14], Wagman et al. [12] }\end{array}$ & Lung (lower lobe) & $\approx 10 \mathrm{~mm}(0-34)$ & $15 \mathrm{~mm}(\mathrm{CC})(5-22)$ \\
\hline Malone et al. [18] & Prostate & $3.3 \mathrm{~mm}(1-11)$ & - \\
\hline Suramo et al. [9], Bryan et al. [19] & Pancreas & $18 \mathrm{~mm}(0-35)$ & $43 \mathrm{~mm}(20-80)$ \\
\hline Suramo et al. [9], Davies et al. [10], Balter et al. [11], Wagman et al. [12] & Kidney & $3 \mathrm{~mm}(2-40)$ & $\approx 20 \mathrm{~mm}(4-86)$ \\
\hline
\end{tabular}

(CC): Craniocaudal direction, $(x-y)$ : min-max amplitude.

As technology advances, other strategies are proposed to reduce the impact of respiratory movements. These new strategies, grouped under the general term of respiratorygated radiotherapy, appear to be promising. Ling showed how technological developments in radiotherapy for lung cancer, including respiratory-gated radiotherapy, should allow dose escalation while maintaining a similar complication rates [20]. As a result, it could improve local control or even overall survival [21]. Regardless of the method used, benefits are expected in terms of geometric precision as well as dosimetric improvements [22-24].

The integration of respiratory movements into treatment planning improves the quality of computed tomography (CT) images and therefore the accuracy of contouring. It is now accepted that the apparent position of intrathoracic organs obtained by a free-breathing CT scan is not representative of an average position between inhalation and exhalation [25]. The use of respiratory gating during the CT simulation session allows the acquisition of the anatomical data and then the irradiation of the target volume in a specific respiratory phase. Theoretically, this technique improves accuracy and reproducibility of treatment. Reducing organ motion with inhale or exhale breath-hold technique has been the subject of several studies $[1,26]$. For example, Hanley et al. have observed, by fluoroscopy on 5 patients, a reproducibility of the diaphragm position between respiratory cycles of $2.5 \mathrm{~mm}$ with inhale breath-hold technique against $26.4 \mathrm{~mm}$ in freebreathing [27]. Nevertheless, even if the motion reduction has been demonstrated, it does not always result in a reduction in safety margins between CTV and PTV due to the lack of confidence in these systems among some teams $[16,28]$.

Forced shallow breathing with abdominal compression was originally developed for stereotactic body radiation therapy (SBRT) of lung and liver tumors at Karolinska Hospital (Stockholm, Sweden) [28-31]. This technique employs a plate that is pressed against the patient's abdomen. The pressure plate is attached to a stereotactic body frame by a rigid arc. Abdominal compression can decrease tumor motion during radiation treatment which can be beneficial for patient with initial motion exceeding $5 \mathrm{~mm}$ [32].

For respiratory gating, two different methods were considered to overcome these unwanted movements, by soliciting participation of the patient or not. Both methods have led to the creation of different techniques either by realtime monitoring of free-breathing or by managing a long and reproducible breath-hold $[3,4,30]$.

In the early 2000s came the Real-time Position Management (RPM) system from Varian Medical System (Palo Alto, USA) consisting of two reflectors attached to an external marker placed on the patient's abdomen. The marker motion, reflecting the breathing pattern of the patient, is analyzed by software that controls the scanner and/or the accelerator, based on predefined criteria $[33,34]$. Other existing real-time tracking devices use different types of mechanical, luminous, or spirometric sensors [1, 30, 35-37].

In the same period appeared spirometers dedicated to the practice of breath-hold: ABC for "active-breathing control" (Elekta, Stockholm, Sweden) and SDX (Dyn'R, Toulouse, France) [38, 39]. Breath-holding, usually in inspiration, is performed during image acquisition and irradiation. It can be obtained by a spirometer connected to a balloon valve (ABC) or by voluntary breath-hold (SDX). These systems were the first to be used routinely. The method was originally proposed by the Memorial Sloan Kettering Cancer Center (MSKCC) team in New York [27, 40, 41]. Objectives and practical implementation differ depending on which of the techniques or strategies are applied [27]. The type of approach is generally driven by the clinical objectives and their adaptations to the patient $[39,42]$.

Another category is tumor tracking, which consists of two major aspects: real-time localization of, and real-time beam adaptation to, a constantly moving tumor $[1,36,37,43]$.

In addition to the improvement in the accuracy to identify the structures and therefore the potential reduction of the margins, the above mentioned techniques would present a potential dosimetric advantage. In breath-hold techniques, the respiratory phase on which irradiation is delivered is selected in order to be the most favorable in terms of distance between the target volume and the critical structures. Therefore, deep-inspiration breath-hold (DIBH) is generally favored, which is close to the maximal respiratory capacity. Thus for a same beam configuration, the lung volume irradiated to a significant dose represents a smaller fraction of the total lung volume. Rosenzweig et al. have shown in a theoretical study, that for the same normal tissue complication probability (NTCP) of $25 \%$, it could be possible 
to increase the prescribed dose from $69.4 \mathrm{~Gy}$ in free-breathing to 87.9 Gy in DIBH [40].

These techniques are presently being investigated in several medical centers worldwide. This paper describes the most frequently used gating and tracking techniques and the main published clinical reports.

\section{Methods and Materials}

2.1. Motion-Encompassing Methods during the CT Simulation Session. An early approach to deal with the uncertainties related to breathing consists of measuring respiratory movements and integrating them into treatment planning. These measurements can be performed either in a representative sample of the general population, or directly on the patient before irradiation. Accumulation of a large data set allows calculation of the statistical distribution of each respiratory movement [42, 44, 45]. In fact, the amplitude of these movements can vary as a function of various factors related to the tumor (tumor site, attachment to the chest wall), the patient (respiratory capacity, oxygenation, anxiety), or the treatment protocol (personalized immobilization, position of the arms).

Once this data has been collected, two approaches are possible. The first approach, most widely used in practice, consists of applying the measured amplitude of respiratory movements to the ITV [46]. The dose prescribed to the PTV is then calculated on a static patient model, by assuming that CTV always remains within the PTV. While this approach, based on the addition of a geometrical margin, has the advantage of being independent of the treatment planning system (TPS), it does not describe the uncertainties in the normal tissue dose distribution.

A second approach is based on modeling the effect of respiratory motion directly in the dose calculations. However, it assumes that the target moves but is not deformed during treatment and that the medium is homogeneous. Determination of the dose-volume histogram (DVH), which takes respiratory movements into account, provides information about degradation of the dose distribution in the target volume and critical organs during breathing and allows estimation of the effects of respiratory motion on TCP and NTCP [47-49].

2.2. Forced Shallow Breathing with Abdominal Compression. To determine if the patient could benefit from this technique, the tumor motion in cranial-caudal direction is generally assessed using a fluoroscopic simulator. If the tumor motion exceeds $5 \mathrm{~mm}$, the abdominal compression is applied [29]. Others use this technique systematically for each patient treated with SBRT who tolerates the abdominal pressure.

The immobilization and positioning is done using a stereotactic body frame that is custom fitted to each patient. A pressure plate is applied to the abdomen and positioned 2 to $3 \mathrm{~cm}$ below the triangular rib cage border. The maximum pressure that is comfortably tolerated by the patient is used during the treatment. The position of the plate is controlled by a scaled screw in order to apply a reproducible amount of abdominal compression $[28,29]$. Daily verification imaging is necessary to ensure respiratory motion reproducibility [50].

2.3. Free-Breathing Gating. These methods involve real-time monitoring of patient free-breathing and trigger the CT scan acquisition and/or linear accelerator at a specific respiratory phase. One of the most widely used gating systems is the RPM. We will take this device as an example, to detail the preparation, CT scan acquisition and treatment delivery phases.

\subsubsection{Gating Devices}

RPM System. The RPM system tracks the respiratory cycles of the patient through a reflective plastic box placed on the patient's abdominal surface. The box must always be installed in the same position, typically midway between the xiphoid process and the umbilicus, which is usually the external region of the body with the greatest breathing motion [32, 51, 52]. The reflectors return light from an infrared illuminator to a Charge-Coupled Device (CCD). This is a camera that occupies a fixed position relative to the patient and is connected to a computer itself linked to the accelerator (Figure 1). The movement of the reflectors induced by breathing is analyzed in real-time by software which controls the triggering of the accelerator, based on a predefined gating window. The beam is then interrupted between each breath and the total dose is delivered in small fractions of a few monitor units. The RPM system is also used to track the breathing cycles while acquiring the CT scan, either to select a predetermined window (prospective mode) or to retrospectively reconstruct respiratory phases (retrospective mode or four-dimensional (4D) CT) [33, 52].

Other Devices. Other gating devices have been tested, often on a few patients only, with different types of mechanical, luminous, or spirometric sensors. The most widely used mechanical system, particularly for imaging purposes, is the gating device proposed by Siemens (Munich, Germany) which consists of acquiring the respiratory signal by a belt (AZ-733V Anzai) equipped with a strain gauge attached directly to the patient. This belt detects the abdominal movements by measuring pressure variations. The analog signal is digitized and sent to the monitoring station and then to the imaging device (CT, magnetic resonance imaging (MRI), positron emission tomography (PET)) or to the accelerator. The software records the patient's respiratory data and controls the CT or irradiation beams accordingly. With the same brand CT, it is also possible to obtain gated images in prospective mode $[16,53,54]$.

2.3.2. Preparation and CT Scan Acquisition. Regardless of which gating device is used (RPM, Anzai, etc.) the preparation for the treatment (immobilization, isocentre selection, etc.) is the same as for conformal radiotherapy (CRT). It seems unnecessary to provide a prior training session, although better breathing pattern reproducibility could be achieved with visual and/or audio coaching [1, 55, 56]. 


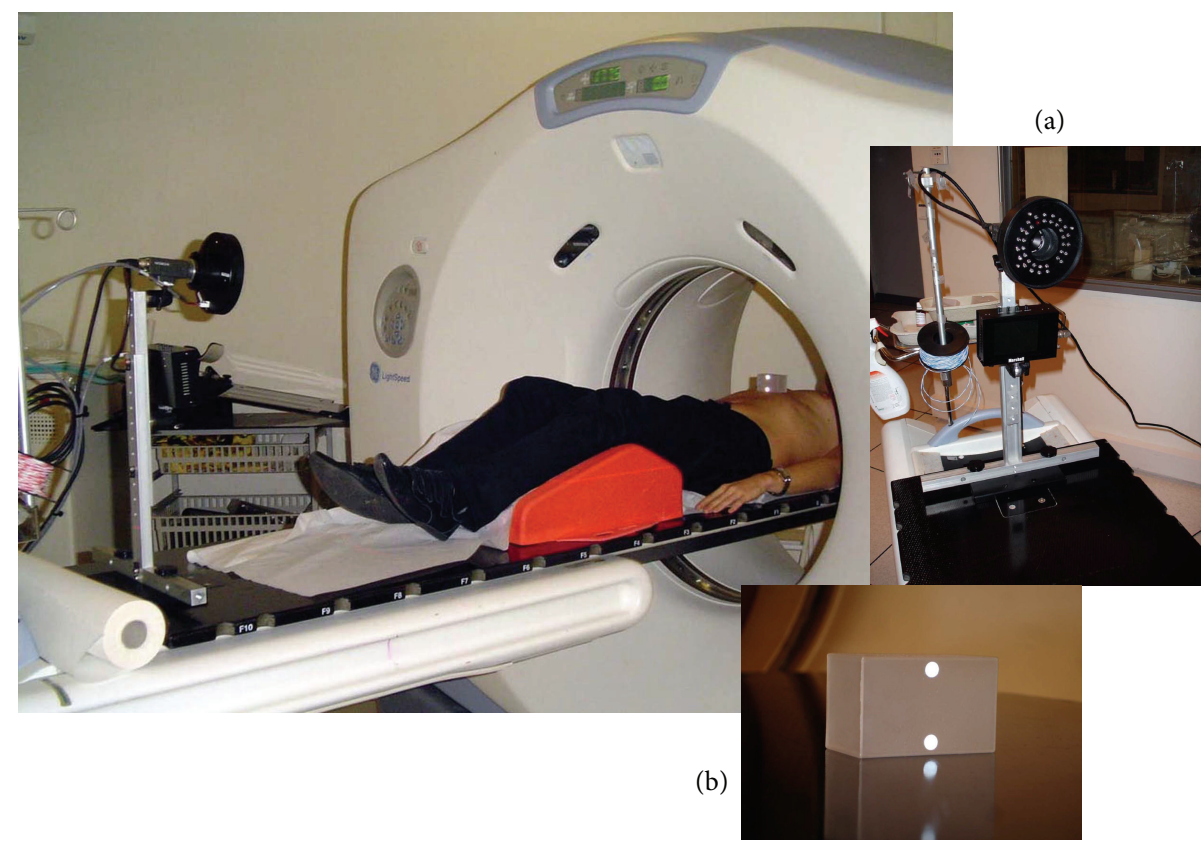

FIGURE 1: The VARIAN RPM system. An infrared camera (a) illuminates a marker on which are attached two reflectors (b). The marker is placed on the patient's abdomen.

The system is based on free-breathing patient, so no specific precaution is necessary regarding skin markers.

By contrast to breath-hold techniques, respiratory-gated techniques in free-breathing require a specific adaptation of the device during image acquisition. It should be a specific software interface that allows communication with an external system responsible for acquisition and analysis of the respiratory signal (RPM external box or belt). Irrespective of the selected mode (retrospective or prospective), in addition to the respiratory signal, the external system records the precise moment when the images are taken. In the case of 4D CT (retrospective), it allows all images of a particular respiratory phase to be reconstructed into a complete threedimensional (3D) CT scan $[15,51,52,55,56]$.

2.3.3. Treatment. Constraints during respiratory-gated radiotherapy are worse in free-breathing than with breath-hold techniques, especially when they are coupled with intensitymodulated radiation therapy (IMRT) [57]. Indeed, the physical limits of this type of irradiation, which is "hyperfractionated" in multiple short exposures, can be problematic [5759]. We must ensure beforehand that the accelerator can go from beam-on to beam-off very quickly while ensuring stable delivery rate, energy and uniformity. The treatment delivery must be done under the same conditions as its preparation. The positioning of the RPM box on the patient skin previously marked during preparation, can seem simple, but it must be ensured that the movements of the external device are not impeded by the patient's accessories or clothing [60]. For quality assurance purposes, it is also important to make sure that there is no phase shift during treatment delivery. A phase shift means that the target position would not match the respiratory monitoring [61].
An important concept in phase-based gating is called gate or gated window. It is a preset phase during the breathing cycle, when the radiation beam will be activated. Typically, the gated window is selected where the residual tumor motion is estimated to be low. Another important concept is the duty cycle, which is a measure of the efficiency of the method. It is the ratio of beam-on time to the total treatment time. For a typical gated CRT the duty cycle is around 30 to $50 \%$ and less than $30 \%$ for gated IMRT. The choice of gate width is a compromise between residual tumor motion and duty cycle [62]. In most of the cases, exhale phase is selected as the gated window, because this is a phase in which breathing movement is reproducible, residual tumor motion is low, and duty cycle longer. On the other hand, during the exhale phase the lung volume is minimal, potentially leading to dosimetric detriment in terms of dose to organs at risk. Session time is extended with phase-based gated-radiotherapy, so it is important to select patients who really benefit from this technique in a dosimetric point of view. A PTV reduction of at least $50 \%$ is achieved on less than $15 \%$ of patients [63]. There is a correlation between the extent of tumor mobility (at least $1 \mathrm{~cm}$ ) and a significant reduction in normal tissue irradiation achieved with gating.

2.4. Breath-Hold. We will take as an example the voluntary DIBH technique (SDX, Dyn'R, Muret, France) to detail the preparation, CT scan acquisition, and treatment phases.

2.4.1. Preparation Training Session. Preparation for the DIBH technique is necessary to provide the technical knowledge and skills in order to foster patient autonomy. The typical training session lasts on average 25 to $55 \mathrm{~min}$, depending on respiratory capacity and understanding of the patient. 


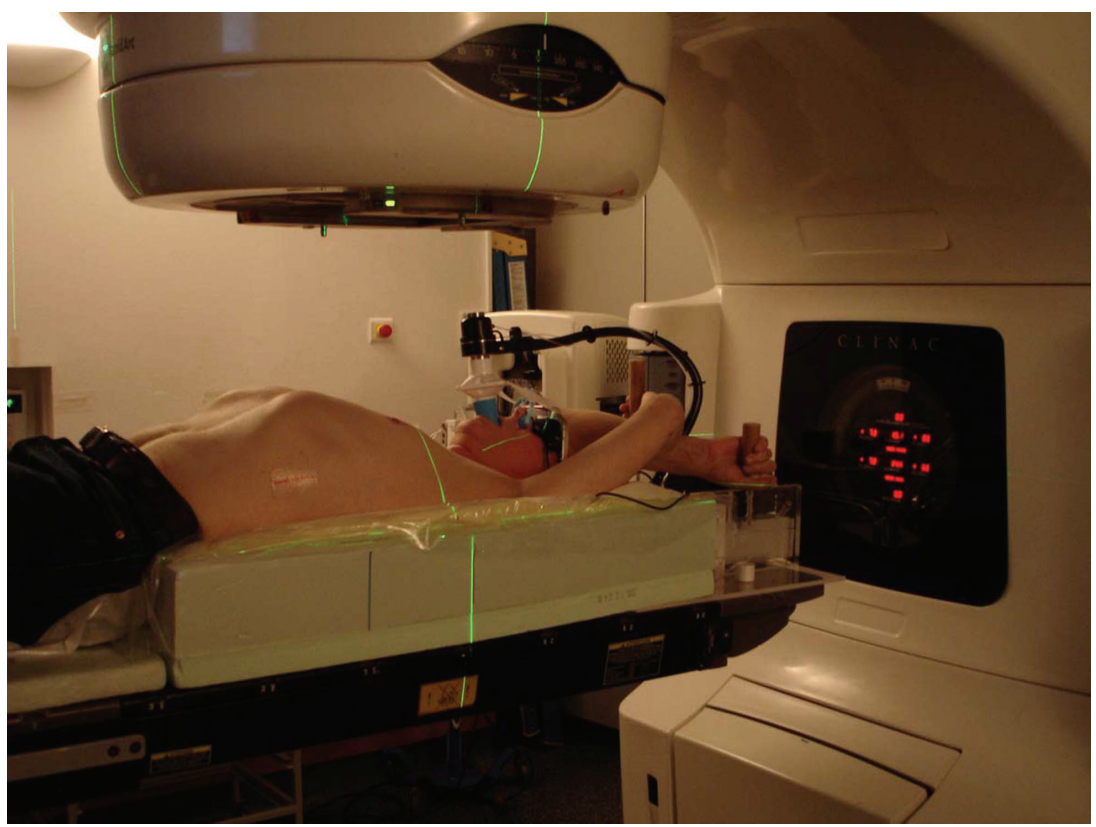

FIgURE 2: Patient in treatment position with SDX (Dyn'R, Toulouse, France) spirometric device, mouthpiece, nose clip, and video goggles.

During this initial contact, the patient should be reassured regarding other available technical possibilities if he was to fail to achieve breath-holding. Indeed, some patients cannot perform the maneuver reproducibly enough to permit its use. Following equipment introduction and explanation on how it works, the first step is to find the most comfortable deep inspiration threshold (Figure 2). It is not possible to apply a standard threshold to all patients. This threshold is also dependent on the clinical objective. It differs between lung, liver, or breast tumors irradiation. To irradiate a lung tumor, we aim at maximum lung expansion, thus reducing the amount of healthy tissue irradiated in proportion to the total lung volume. For breast cancer, especially on the left side, deep inhalation allows partial or total exclusion of the heart from the beam. Hepatic lesion irradiation essentially requires immobilization of the liver. In addition to these clinical considerations, the overall condition of the patient must be taken into account. In all cases, the deep inhalation should be restricted to an effortless thoracic expansion $[1,39$, 42, 64-66].

For this first training phase, the patient must performs several (3-4) short breath-holds, initially without the video coaching, and then deeper inspiration based on his own sensations. The optimal inspiration threshold is selected based on these exercises. It is considered that from a 15 seconds breath-hold, CT image acquisition and irradiation become feasible, knowing that it is possible to deliver the radiation treatment on several breath-holds. The second part of the learning phase is to train the patient in the same condition as during CT scan acquisition or treatment delivery. The patient observes his own breathing trace in the goggles screen and maintains the amplitude within the prescribed visual limit (Figure 3) [60, 64, 67]. The first method proposed by MSKCC team in New York was to give audio prompting using the intercom, but the audio coaching was found to be significantly more difficult to follow than visual feedback $[27,40,41,68]$.

2.4.2. CT Scan Acquisition. The CT scan acquisition takes place with the same inspiration threshold as during the training session. However, it is still possible to refine this threshold at this point. If iodine contrast is required, it is necessary to adapt the beginning of this injection and the duration of the infusion according to the breath-hold and to the purpose of the infusion [60].

2.4.3. Treatment. Treatment sessions are conducted by successive request to the patient to hold inhalation to the predetermined threshold, during each verification imaging or radiation beam. If the instructions during the training and the previous radiation session put the patient at ease, the beams sequence is easily achieved $[40,41]$.

2.5. Real-Time Tumor-Tracking. Another category of methods for managing respiratory motion during the treatment is tumor-tracking, which consists of two major aspects: realtime localization of, and real-time beam adaptation to, a constantly moving tumor. Compared to the motion freezing methods, tumor-tracking techniques potentially offer additional benefits such as higher delivery efficiency and less residual target motion. These factors may be particularly important in radiosurgery of thoracic and abdominal tumor sites, where a large dose is delivered during a single relatively lengthy treatment session. Delivering a large dose at one time requires high dose conformity to the target, and the lengthy treatment time demands a high degree of dose delivery efficiency and a procedure that is comfortable to patients. It 


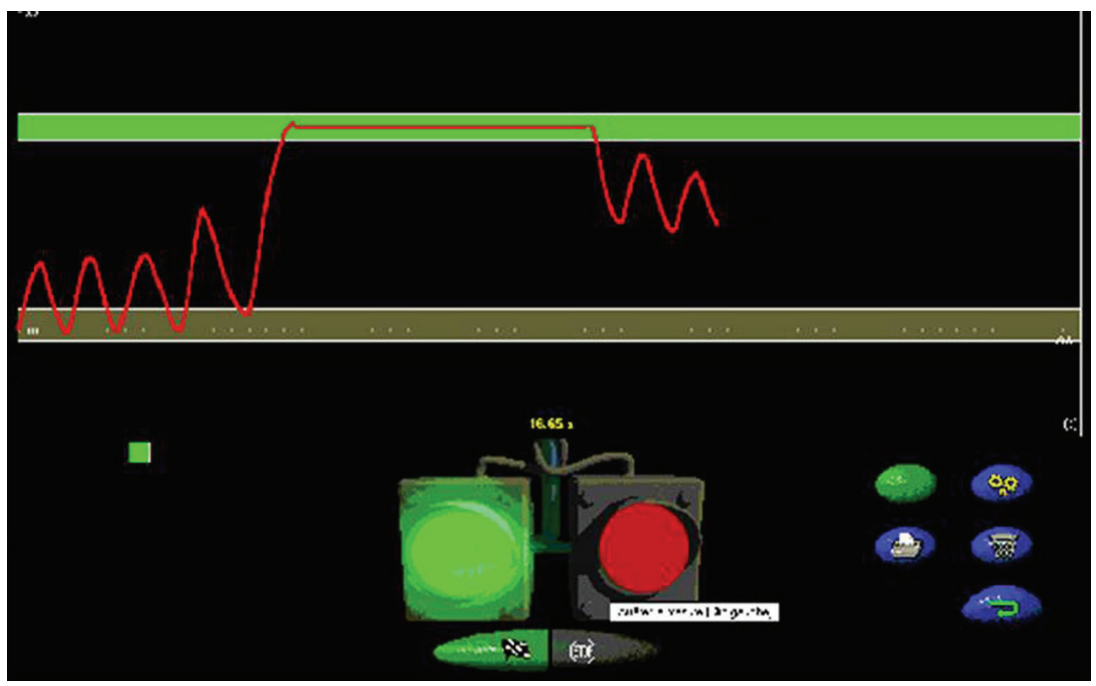

FIGURE 3: Diagram of the respiratory trace visualized by the patient in goggles screen and the therapist with the SDX device during a self-held breath-hold. The patient sees the evolution of his lung volume over time (yellow curve) and its breathing amplitude (green zone) predetermined during the training session. After three cycles of free-breathing, the patient is asked to reach this amplitude and to maintain its breath-hold (yellow plateau of the curve). During this period the CT scan acquisition or irradiation is done. He then resumed his normal breathing.

is important to note that real-time beam adaptation is not feasible without precise real-time localization of the tumor position in $3 \mathrm{D}$.

\subsubsection{Real-Time Tumor-Localization}

The Use of External Respiratory Surrogates. In most gating techniques, tumor position is extrapolated from surrogate breathing signals such as lung volume or skin motion. The short term correlation between external surrogates and internal target position may be high for some tumor sites; however, the correlation may be not stable during a long treatment fraction, owing to transient changes in breathing and waveform drifts [56, 69]. In addition, the internalexternal correlation may change over the treatment course. The diaphragm may be used as a surrogate landmark for lower lobe lung, liver, and pancreas tumors, if fluoroscopy is available during treatment [15, 69-72]. Again, the correlation between diaphragm and target position may not hold for a long treatment time and from fraction to fraction. Therefore, the prediction of tumor position from external surrogates should be used with great caution. If one decides to use this approach, the correlation between surrogate and tumor position should be established before each treatment fraction and be checked/updated during the treatment session at a frequency depending on the tumor site or even the individual patient [56]. The major advantage of this approach is the reduced radiographic dose.

The Use of Implanted Radio-Opaque Fiducial Markers. High$\mathrm{Z}$ metal fiducial markers can be implanted in the tumorbearing organs to help localize the tumor position in realtime. Spherical or cylindrical gold markers are often used for this purpose $[56,73,74]$. Markers can be implanted either percutaneously or endoscopically, depending on the tumor location and other medical considerations. The high radioopacity of the markers makes them readily detectable in fluoroscopic images. Marker positions can be calculated through a simple and fast triangulation process. Therefore, fiducial marker based tumor localization is relatively straightforward from the image processing point of view and can be done in a very efficient way to facilitate real-time tumor-tracking.

Fiducial marker based real-time tumor-localization has been extensively used at Hokkaido University in Japan for gated treatment of lung, liver, prostate, and other tumor sites [75-78]. Markers are tracked at the video frame rate of $30 \mathrm{~Hz}$ using the Real-time Tumor-Tracking Radiation Treatment (RTRT) system developed by Mitsubishi and Hokkaido University. The RTRT imaging system consists of four $\mathrm{kV}$ X-ray units and is described in more detail in a later subsection.

Percutaneously implanting fiducial markers is an invasive procedure with potential risks of infection. Many clinicians are reluctant to use this procedure for lung cancer treatment because puncturing of the chest wall may cause pneumothorax. The effectiveness of using fiducial markers for tumor localization relies on the stability of the relationship between markers and tumor center. This relationship may change during the treatment course due to changes in tumor geometry. Additionally, markers may migrate within the tissue between the planning CT study and the treatment delivery. For these reasons, three to four markers are often implanted and any marker migration detected by monitoring the inter-marker spacing. Three markers also allow detection of tumor rotation [43].

Nonradiographic Tumor-Tracking. Efforts have been made to track tumors nonradiographically. A miniature, implantable radiofrequency (RF) coil has been developed by 
Seiler et al. that can be tracked magnetically in 3D from outside the patient [77]. A new technology currently under development at Calypso Medical Technologies, Inc., Seattle (USA), is based on non-ionizing electromagnetic fields, using small wireless transponders implanted in human tissue [53]. Real-time 3D ultrasound is another possible alternative to X-ray imaging for tracking the tumor [79].

Direct Tracking of Lung Tumor Mass. Owing to the risk of pneumothorax, percutaneous implantation of fiducial markers should be avoided whenever the lung tumor can be tracked directly. Direct fluoroscopic tumor tracking is extremely difficult, if not impossible, for tumors in abdomen. In the case of lung tumors, however, the density difference between the tumor mass and normal lung tissue may be large enough to provide a good visualization in radiographic images. Berbeco et al. have found that direct detection of a lung tumor in $\mathrm{kV} \mathrm{X}$-ray images is possible if the tumor mass is small, well-defined, and has a high-contrast edge [80]. Early stage lung cancer patients may have tumors that fulfill these requirements, and those patients may benefit from extracranial radiosurgery based on precise tumor tracking. For cases where contrast between tumor and normal tissue is low, direct tumor-tracking may still be feasible, if advanced image processing/computer vision techniques are used. This technology is still in an early investigational stage.

\subsubsection{Real-Time Beam Adaptation}

Methods for Real-Time Beam Alignment. By knowing the tumor position during the treatment, through real-time marker tracking and position predicting, the treatment delivery system can respond accordingly. One way is to gate the beam-on at a particular tumor position; the other is to align the beam with the instantaneous tumor position [74]. Murphy has summarized four possible ways for real-time beam alignment: (1) move the patient using a remotely-controlled couch, (2) move a charged particle beam electromagnetically, (3) move a robotically mounted lightweight linear accelerator, and (4) move the aperture shaped by a dynamic multileaf collimator (DMLC) [43].

Technically, it is feasible to shift the patient to cancel out the tumor motion by repositioning a remote-controlled couch [81]. However, to track respiratory motion, the practicality of this method is questionable, because the constant motion will cause problems of patient comfort and the nonrigidity of the human body will compromise the tracking accuracy. Within the context of conventional X-ray radiotherapy, we will address methods 3 and 4 for real-time beam alignment.

The CyberKnife system has implemented motion of a linear accelerator in real-time to follow the tumor, by means of a lightweight $6 \mathrm{MV} \mathrm{X}$-band linac mounted on an industrial robotic arm. A real-time control loop monitors the tumor position from the imaging system and directs the repositioning of the linac $[56,73,82]$. The major strength of the system is that it can move and orient the X-ray beam with six degrees of freedom, so that it can adapt to the full 3D motion of the tumor. A disadvantage is that the system has limited beam output and beam size, therefore the treatment time can be lengthy for large size tumors.

Tumor tracking by means of a DMLC shaped aperture is an active area of investigation [83-90]. DMLC has become a standard means of IMRT delivery on some gantry-mounted linacs. The MLC leaf travel speed can safely reach $2.5 \mathrm{~cm} / \mathrm{s}$, which is comparable with breathing induced tumor motion speed. Since it only moves the beam aperture in two dimensions, the approach cannot compensate out-of-plane tumor motion. However, the resultant dosimetric error should be small.

Because of its potential for providing high dose conformity and high duty cycle, as well as its technical complexity, real-time beam adaptation methods are suitable for hypofractionated thoracic and abdominal cancer. The actual tumor movement as well as its relationship to surrounding critical structures during the treatment cannot be known at the time of treatment planning. Therefore, treatment planning can only be done based on some kind of average patient geometry information or at best on $4 \mathrm{D}$ CT simulation data, and an adaptive scheme must be used throughout the treatment course.

\section{Results}

3.1. Forced Shallow Breathing with Abdominal Compression. Negoro et al. reported that abdominal compression can lead to a significant reduction in tumor motion [32]. Tumor motion was assessed for 18 patients with a solitary lung tumor. Eleven of 18 patients presented a respiratory tumor movement greater than $5 \mathrm{~mm}$, so they underwent CRT using abdominal compression. Daily portal imaging was used for patient alignment. Setup error tolerance was $3 \mathrm{~mm}$, requiring repositioning in $25 \%$ of the daily setups. For 10 patients, the range of motion before abdominal compression was 8 to $20 \mathrm{~mm}$ (mean: $12.3 \mathrm{~mm}$ ), reduced to 2 to $11 \mathrm{~mm}$ (mean: $7.0 \mathrm{~mm}$ ) with compression $(P=0.0002)$. For one patient, the pressure plate was not used, because respiratory motion had paradoxically increased.

Interfraction target deviation has been analyzed for patients undergoing liver or lung SBRT with abdominal compression [91]. The standard deviation in all directions is generally lower than $5 \mathrm{~mm}$. However, some studies stated that this technique could possibly present reproducibility issues. Interfraction and intrafraction changes in amplitude of target motion during SBRT were assessed with cone beam computed tomography (CBCT). It shows that abdominal compression causes larger variations in the time spent on the treatment couch and in the inter- and intrafraction changes in tumor motion amplitude $[12,92,93]$. Nevertheless, Wunderink et al. analyzed liver tumor motion variations for patients treated with abdominal compression in stereotactic body frame, by tracking fiducial markers. They concluded that the residual motion had a good reproducibility during the treatment course and that cranial-caudal excursions measured on the treatment days were never significantly greater than on the planning days [94]. 
3.2. Free-Breathing Gating. Up until now, few studies have evaluated the various devices in free-breathing. Berson et al. evaluated the RPM system on 108 patients irradiated for diverse tumors (lung, liver, breast, mediastinum) [64]. Ninety-seven patients were able to be completely treated with this device with an average session time multiplied by 4 . Ford et al. have used this technique on 8 patients treated for lung or liver tumors [15]. Residual intra- and interfractions movements measured by fluoroscopy and portal imaging were, respectively, evaluated to $2.6 \pm 1.7 \mathrm{~mm}$ and $2.8 \pm 1.0 \mathrm{~mm}$. Using audio coaching or "biofeedback" techniques, via screen or video goggles to inform the patient in real time on its position in the respiratory cycle, has significantly reduced the residual movements and eliminated part of the synchronization errors $[15,67]$. The same team from MSKCC has evaluated this technique on 10 patients with hepatocellular carcinoma [12]. Only 8 out of these 10 patients have been actually irradiated with the RPM system, all in exhalation phase, representing 177 respiratory-gated treatments. Using this technique, movements of the diaphragm in the cranialcaudal direction have been reduced by an average of $22.7 \pm$ $7.4 \mathrm{~mm}$ without respiratory gating to $5.1 \pm 2.1 \mathrm{~mm}$ with it. The difference of the position of the diaphragm between the digitally reconstructed radiograph (DRR) and the portal imaging has been on average $2.3 \mathrm{~mm}(0,1,10,8)$. Reducing respiratory motion of the liver has reduced safety margins of $1 \mathrm{~cm}$ (total margin of $1 \mathrm{~cm}$ instead of $2 \mathrm{~cm}$ initially) and, consequently, authorized an increase in the total dose of 7 to $27 \%$ depending on the patient (median: 21.3\%) [12]. Underberg et al. analyzed 4D CT scans on 31 patients for a total of 34 lung tumors [63]. The mean mobility vector was decreased from $8.5 \pm 6.5 \mathrm{~mm}$ for the entire breathing cycle to $1.4 \pm 0.7 \mathrm{~mm}$ in the tidal-expiration gated window. For the 34 tumors, 3 PTVs were created: PTV 1 bins (ITV from 4D

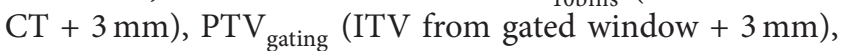
and $\mathrm{PTV}_{10 \mathrm{~mm}}(\mathrm{GTV}+10 \mathrm{~mm})$. PTV $10 \mathrm{bins}$ and $\mathrm{PTV}_{\text {gating }}$ were on average $48 \%$ and $33 \%$ of $\mathrm{PTV}_{10 \mathrm{~mm}}$ and mean volumes of normal tissue receiving the prescribed doses were $57 \%$ and $39 \%$ of PTV $_{10 \mathrm{~mm}}$, respectively.

3.3. Spirometric Monitoring of Breathing Pattern. Breath-hold devices, voluntary or not, were the first to be used and evaluated. Wong et al., in one of the original studies, have used the $\mathrm{ABC}$ system on 12 patients treated for lung, liver cancer or Hodgkin's disease [38]. Breath-hold was directed only at early-expiratory or end-inspiratory phase for an average of 10 to 15 seconds. Two to three breath-holds were sometimes necessary to deliver a full radiation session. Measured on only 5 patients, residual intra- and inter-fractions movements of the diaphragm were, respectively, $1.5 \pm 1.8 \mathrm{~mm}$ and $4.0 \pm$ $3.3 \mathrm{~mm}$ [38]. Remouchamps et al. evaluated the same ABC system for breast irradiation [21]. Fifteen patients, 9 left and 6 right breast cancers, were treated with this technique with good tolerance. The active-breathing control with inhalation breath-hold by decreasing respiratory motion of the breast target volume helped to reduce the size of the ITV from 15 to $5 \mathrm{~mm}$. As a result, the cardiac volume receiving at least $30 \mathrm{~Gy}$ $\left(\mathrm{V}_{30}\right)$ and the lung volume receiving at least $20 \mathrm{~Gy}\left(\mathrm{~V}_{20}\right)$ have been significantly reduced. This reduction was even greater when treating with IMRT: the $\mathrm{V}_{30}$ and $\mathrm{V}_{20}$ decreased from 19 to $3 \%$ and 24 to $15 \%$, respectively [21]. Rosenzweig et al. assessed one of the first breath-hold devices called "DIBH" on 7 patients irradiated for a nonsmall cell lung cancer (NSCLC) [40]. Each breath-hold lasted 12 to 16 seconds and could be repeated without fatigue from 10 to 13 times per session. Residual intrasession and intersession diaphragmatic movements were, respectively, $1 \pm 9 \mathrm{~mm}$ and $2.5 \pm 1.6 \mathrm{~mm}$. Reduction of respiratory motion allowed for the decrease of the ITV margins from $1-2 \mathrm{~cm}$ to $0.2-0.5 \mathrm{~cm}$ bringing a $30 \%$ reduction in the volume of healthy lung receiving at least $25 \mathrm{~Gy}\left(\mathrm{~V}_{25}\right)$. Theoretical reduction in the risk of radiation pneumonitis could allow for the increase of the total dose from 69.4 to $87.9 \mathrm{~Gy}$ [40]. Kim et al. studied on 16 patients the reproducibility of this technique at different respiratory phases [93]. The best reproducibility was observed in deep hold inspiration and expiration. Under these circumstances, the mean residual motion of the diaphragm was $0.97 \mathrm{~mm}$ ( 0.13 to $2.57 \mathrm{~mm}$ ) during one session, and less than $5 \mathrm{~mm}$ on the whole treatment ( 35 sessions).

3.4. Prospective Clinical Results. In 2003, a medicoeconomic assessment of the various breathing-adapted radiotherapy methods for the treatment of lung cancers was conducted in the context of a project funded by the French Ministry of Health, entitled Programme de Soutien aux Innovations Diagnostiques et Thérapeutiques Coûteuses (STIC) (Expensive Diagnostic and Therapeutic Innovation Support Program). Twenty radiotherapy departments from all over France, specializing in the treatment of lung cancer, participated in this joint project to guide decision-makers and to provide precise data for or against the diffusion of this innovation by comparing it with the standard treatment: CRT without respiratory gating. This study was initiated in April 2004 according to a nonrandomized design, but this does not constitute a limitation, as this study was based on a pragmatic approach. At the end of the recruitment phase, 668 patients were included: 319 patients in the respiratory-gated radiotherapy group (RGRT) and 349 in the reference group (CRT). This is the largest study to date evaluating the various breathingadapted radiotherapy techniques to optimize irradiation of lung and breast cancers $[95,96]$.

Concerning breast cancer (233 patients), radiation treatment is effective and there is a low rate of toxicity. The very low number of events in terms of toxicity and relapse limits the comparison between CRT and RGRT techniques. The first important outcome of this part of the study concerns the good reproducibility of the measured total lung volume from the different gating systems. The second point concerns the higher total lung volume in the RGRT group with a corresponding decrease in predictive parameters for pulmonary toxicity. This benefit is logically more important for the 2 systems in maximal inspiration (ABC and SDX) compared to the RPM system (on average additional $895 \mathrm{~mL}$ for total lung volume; $2334 \pm 409 \mathrm{~mL}$ versus $1438 \pm 443 \mathrm{~mL}, P<0.00001)$. This significant increase in healthy lung volume has led to a significant reduction of dosimetric parameters: $\mathrm{V}_{25}(5.5 \%$ versus $6.6 \%, P<0.0001), \mathrm{V}_{37}(3.8 \%$ versus $4.8 \%, P<0.0001)$, and mean dose (3.9 Gy versus $4.4 \mathrm{~Gy}, P<0.005)$, respectively, 
between breath-hold and free-breathing techniques. The last point concerns the trend with the breath-hold technique towards a decrease in the dose to the contralateral breast. Specifically, there is an interest regarding the different breathhold techniques when treating the left breast in order to protect the heart (using this technique, the maximum dose to the heart is decreased by an average of $11 \mathrm{~Gy}$ for the left breast compared with $2 \mathrm{~Gy}$ for the right breast $(P<0.0001))$ [95].

For lung cancer (435 patients), the treatment is unfortunately limited in terms of efficiency and is relatively toxic. The large number of local relapse within the radiation fields associated with significant pulmonary and esophageal toxicity confirms the need for a highly accurate technique to increase the dose in the target volume while protecting the surrounding healthy tissue. The breath-hold method could help to achieve those objectives. As for breast cancer, the first important result from this part of the study shows the good reproducibility of the different gating systems. The second point also concerns the higher total lung volume in the RGRT group with a corresponding decrease in predictive parameters for pulmonary toxicity. This benefit is a lot more important for the 2 systems in maximal inspiration ( $A B C$ and SDX) compared to the RPM system (on average additional $1421 \mathrm{~mL}$ for total lung volume; $5371 \pm 1485 \mathrm{~mL}$ versus $3949 \pm$ $1272 \mathrm{~mL}, P<0.00001)$. It is resulting in a significant reduction of dosimetric parameters such as the $\mathrm{V}_{20}(22.8 \%$ versus $26.5 \%, P<0.0001), \mathrm{V}_{25}(18.8 \%$ versus $23.2 \%, P<0.0001)$, $\mathrm{V}_{37}(11.8 \%$ versus $15.1 \%, P<0.0001)$ and the mean dose (12.8 Gy versus $15.6 \mathrm{~Gy}, P<0.0001$ ), respectively, between breath-hold and free-breathing techniques. Specifically in the breath-hold subgroup, this dosimetric benefit was correlated with a decrease in acute and late pulmonary toxicity observed clinically and functionally (pulmonary function tests). This reduction in toxicity through different gating techniques was also observed for the heart and the esophagus (significant reduction of $\mathrm{V}_{40}$ : $8.1 \%$ versus $11.5 \%, P<0.0001$ ). On the other hand, there was no difference in terms of efficiency (overall and specific survivals) depending on the techniques [96].

\section{Discussion}

The different respiratory gating methods lead to a real clinical benefit. Their comparison has demonstrated that they each have their specificities $[1-3,64,97-102]$ and their interests in the various situations encountered on a daily basis. The deep breath-hold methods seem to be better established than the free-breathing gating methods when we consider the large number of centers who are currently using it all around the world [1]. However, the effectiveness of the treatment with this method depends on the investment of professionals in coordination with patients because it requires involvement and active cooperation of the patient. Regardless of the technique used, preparation and acquisition phases are of major importance as they create the conditions of their appropriation by each patient $[42,103-108]$.

The STIC project, based on a large number of patients with a long follow-up, confirms the preliminary results published on the various respiratory gating devices derived from smaller patient series $[14,38,40,41,109]$. The primary objective of this study was to compare the clinical and economic aspects of RGRT, an innovative technique proposed to limit the impact of respiratory movements during irradiation, versus CRT. The final results based on 668 evaluable patients confirm the feasibility and good reproducibility of the various respiratory gating systems, regardless of tumor site. The results of this study demonstrated a marked reduction of dosimetric parameters predictive of pulmonary, cardiac, and esophageal toxicity as a result of the various respiratory gating techniques. These dosimetric benefits were mainly observed with DIBH techniques (ABC and SDX systems), which markedly increased the total lung volume compared to the inspiration-synchronized system based on tidal volume (RPM). With a median follow-up of 25 months, these theoretical dosimetric benefits were correlated clinically with a significant reduction of acute and late toxicity, especially pulmonary toxicity. Pulmonary function parameters, especially DLCO, although more heterogeneous, showed a tendency to reduction of pulmonary toxicity in the RGRT group $[95,96]$.

Published studies comparing the various respiratory gating methods show that they all provide a real clinical benefit and that each technique has its own specificities $[1,67,92$, 93] and indications in the various situations encountered in routine clinical practice. However, DIBH techniques are more widely used throughout the world than synchronized techniques [27, 40, 41, 68, 96, 109, 110].

However, the use of RGRT requires additional resources in terms of patient preparation and teaching of deep inspiration breath-hold techniques, and especially in terms of treatment sessions which are on average of several minutes longer than conventional techniques, resulting in increased demands on personnel and equipment $[1,14,86,111,112]$.

All respiratory gating methods also benefit from the contribution of image-guided radiotherapy $[56,62,111]$. Just like bone alignment techniques, visualization of the real position of the target volume during breathing improves the overall quality of radiation therapy. Although the implementation of one or more respiratory gating techniques was initially motivated by the need to limit respiratory movements, these techniques now allow new modalities of irradiation, such as hypofractionated and intensity modulated radiotherapy [113116]. Sophisticated and more or less dedicated apparatuses, such as Novalis TX (BrainLAB, Feldkirchen, Germany), Cyberknife (Accuray, Sunnyvale, USA), or dynamic arc therapy, combined with an appropriate respiratory gating technique are already operational. Tomotherapy (Tomotherapy, Madison, USA) should also be available in the near future [1] .

\section{Conclusion}

Respiratory gating techniques benefit from many developments and studies that have contributed to create the conditions for their routine implementation. However, appropriate methods customized to each patient must be developed. Respiratory gating devices seem to be essential to reduce the acute and late toxicity, including pulmonary, cardiac, and esophageal, during pulmonary radiation therapy. Voluntary or active DIBH appears to be, at least from the dosimetric 
standpoint, more efficient in reducing toxicities than gating systems treating with lung inflation around the tidal volume.

\section{References}

[1] P. Giraud, E. Yorke, S. Jiang, L. Simon, K. Rosenzweig, and G. Mageras, "Reduction of organ motion effects in IMRT and conformal $3 \mathrm{D}$ radiation delivery by using gating and tracking techniques," Cancer/Radiotherapie, vol. 10, no. 5, pp. 269-282, 2006.

[2] K. M. Langen and D. T. Jones, "Organ motion and its management," International Journal of Radiation Oncology, Biology and Physics, vol. 50, no. 1, pp. 265-278, 2001.

[3] M. Goitein, "Organ and tumor motion: an overview," Seminars in Radiation Oncology, vol. 14, no. 1, pp. 2-9, 2004.

[4] F. Reboul, L. Mineur, J. B. Paoli, V. Bodez, R. Oozeer, and R. Garcia, "Thoracic radiotherapy and breath control: current prospects," Cancer/Radiotherapie, vol. 6, no. 1, pp. 135s-139s, 2002.

[5] C. G. Willett, R. M. Linggood, M. A. Stracher et al., "The effect of the respiratory cycle on mediastinal and lung dimensions in Hodgkin's disease. Implications for radiotherapy gated to respiration," Cancer, vol. 60, no. 6, pp. 1232-1237, 1987.

[6] K. Ohara, T. Okumura, M. Akisada et al., "Irradiation synchronized with respiration gate," International Journal of Radiation Oncology, Biology and Physics, vol. 17, no. 4, pp. 853-857, 1989.

[7] ICRU REPORT 83, "Prescribing, recording, and reporting. Photon-beam intensity-modulated radiation therapy (IMRT)," Journal of the ICRU, vol. 10, no. 1, 2010.

[8] J. Chavaudra and A. Bridier, "Definition of volumes in external radiotherapy: ICRU reports 50 and 62," Cancer/Radiotherapie, vol. 5, no. 5, pp. 472-478, 2001.

[9] I. Suramo, M. Paivansalo, and V. Myllyla, "Cranio-caudal movements of the liver, pancreas and kidneys in respiration," Acta Radiologica-Series Diagnosis, vol. 25, no. 2, pp. 129-131, 1984.

[10] S. C. Davies, A. L. Hill, R. B. Holmes, M. Halliwell, and P. C. Jackson, "Ultrasound quantitation of respiratory organ motion in the upper abdomen," The British Journal of Radiology, vol. 67, no. 803, pp. 1096-1102, 1994.

[11] J. M. Balter, K. L. Lam, C. J. McGinn, T. S. Lawrence, and R. K. Ten Haken, "Improvement of CT-based treatment-planning models of abdominal targets using static exhale imaging," International Journal of Radiation Oncology, Biology and Physics, vol. 41, no. 4, pp. 939-943, 1998.

[12] R. Wagman, E. Yorke, E. Ford et al., "Respiratory gating for liver tumors: use in dose escalation," International Journal of Radiation Oncology, Biology and Physics, vol. 55, no. 3, pp. 659668, 2003.

[13] S. Shimizu, H. Shirato, K. Kagei et al., "Impact of respiratory movement on the computed tomographic images of small lung tumors in three-dimensional (3D) radiotherapy," International Journal of Radiation Oncology, Biology and Physics, vol. 46, no. 5, pp. 1127-1133, 2000.

[14] P. Giraud, Y. de Rycke, B. Dubray et al., "Conformal radiotherapy (CRT) planning for lung cancer: analysis of intrathoracic organ motion during extreme phases of breathing," International Journal of Radiation Oncology, Biology and Physics, vol. 51, no. 4, pp. 1081-1092, 2001.

[15] E. C. Ford, G. S. Mageras, E. Yorke, K. E. Rosenzweig, R. Wagman, and C. C. Ling, "Evaluation of respiratory movement during gated radiotherapy using film and electronic portal imaging," International Journal of Radiation Oncology, Biology and Physics, vol. 52, no. 2, pp. 522-531, 2002.

[16] E. A. Barnes, B. R. Murray, D. M. Robinson, L. J. Underwood, J. Hanson, and W. H. Y. Roa, "Dosimetric evaluation of lung tumor immobilization using breath hold at deep inspiration," International Journal of Radiation Oncology, Biology and Physics, vol. 50, no. 4, pp. 1091-1098, 2001.

[17] L. Ekberg, O. Holmberg, L. Wittgren, G. Bjelkengren, and T. Landberg, "What margins should be added to the clinical target volume in radiotherapy treatment planning for lung cancer?" Radiotherapy and Oncology, vol. 48, no. 1, pp. 71-77, 1998.

[18] S. Malone, J. M. Crook, W. S. Kendal, and J. Szanto, "Respiratory-induced prostate motion: quantification and characterization," International Journal of Radiation Oncology, Biology and Physics, vol. 48, no. 1, pp. 105-109, 2000.

[19] P. J. Bryan, S. Custar, J. R. Haaga, and V. Balsara, "Respiratory movement of the pancreas: an ultrasonic study," Journal of Ultrasound in Medicine, vol. 3, no. 7, pp. 317-320, 1984.

[20] C. C. Ling, E. Yorke, H. Amols et al., "High-tech will improve radiotherapy of NSCLC: a hypothesis waiting to be validated," International Journal of Radiation Oncology, Biology and Physics, vol. 60, no. 1, pp. 3-7, 2004.

[21] V. M. Remouchamps, F. A. Vicini, M. B. Sharpe, L. L. Kestin, A. A. Martinez, and J. W. Wong, "Significant reductions in heart and lung doses using deep inspiration breath hold with active breathing control and intensity-modulated radiation therapy for patients treated with locoregional breast irradiation," International Journal of Radiation Oncology, Biology and Physics, vol. 55, no. 2, pp. 392-406, 2003.

[22] T. Bortfeld, S. B. Jiang, and E. Rietzel, "Effects of motion on the total dose distribution," Seminars in Radiation Oncology, vol. 14, no. 1, pp. 41-51, 2004.

[23] J. Mechalakos, E. Yorke, G. S. Mageras et al., "Dosimetric effect of respiratory motion in external beam radiotherapy of the lung," Radiotherapy and Oncology, vol. 71, no. 2, pp. 191-200, 2004.

[24] H. Shirato, Y. Seppenwoolde, K. Kitamura, R. Onimura, and S. Shimizu, "Intrafractional tumor motion: lung and liver," Seminars in Radiation Oncology, vol. 14, no. 1, pp. 10-18, 2004.

[25] P. Giraud, S. Helfre, V. Servois et al., "Evaluation of intrathoracic organs mobility using CT gated by a spirometer," in Proceedings of the 19th ESTRO, Istanbul, Turkey, 2000.

[26] E. D. Yorke, L. Wang, K. E. Rosenzweig, D. Mah, J. B. Paoli, and C. S. Chui, "Evaluation of deep inspiration breath-hold lung treatment plans with Monte Carlo dose calculation," International Journal of Radiation Oncology, Biology and Physics, vol. 53, no. 4, pp. 1058-1070, 2002.

[27] J. Hanley, M. M. Debois, D. Mah et al., "Deep inspiration breathhold technique for lung tumors: the potential value of target immobilization and reduced lung density in dose escalation," International Journal of Radiation Oncology, Biology and Physics, vol. 45, no. 3, pp. 603-611, 1999.

[28] J. Leong, "Implementation of random positioning error in computerised radiation treatment planning systems as a result of fractionation," Physics in Medicine and Biology, vol. 32, no. 3, pp. 327-324, 1987.

[29] H. Blomgren, I. Lax, I. Naslund, and R. Svanstrom, "Stereotactic high dose fraction radiation therapy of extracranial tumors using an accelerator. Clinical experience of the first thirty-one patients," Acta Oncologica, vol. 34, no. 6, pp. 861-870, 1995. 
[30] J. P. Bissonnette, K. N. Franks, T. G. Purdie et al., "Quantifying interfraction and intrafraction tumor motion in lung stereotactic body radiotherapy using respiration-correlated cone beam computed tomography," International Journal of Radiation Oncology, Biology and Physics, vol. 75, no. 3, pp. 688-695, 2009.

[31] I. Lax, "Target dose versus extratarget dose in stereotactic radiosurgery," Acta Oncologica, vol. 32, no. 4, pp. 453-457, 1993.

[32] Y. Negoro, Y. Nagata, T. Aoki et al., "The effectiveness of an immobilization device in conformal radiotherapy for lung tumor: reduction of respiratory tumor movement and evaluation of the daily setup accuracy," International Journal of Radiation Oncology, Biology and Physics, vol. 50, no. 4, pp. 889898, 2001.

[33] P. Keall, "4-dimensional computed tomography imaging and treatment planning," Seminars in Radiation Oncology, vol. 14, no. 1, pp. 81-90, 2004.

[34] P. Giraud, L. Simon, M. Saliou et al., "Respiratory gated radiotherapy: the $4 \mathrm{D}$ radiotherapy," Bulletin du Cancer, vol. 92, no. 1, pp. 83-89, 2005.

[35] T. Tada, K. Minakuchi, T. Fujioka et al., "Lung cancer: intermittent irradiation synchronized with respiratory motion-results of a pilot study," Radiology, vol. 207, no. 3, pp. 779-783, 1998.

[36] G. S. Sharp, S. B. Jiang, S. Shimizu, and H. Shirato, "Prediction of respiratory tumour motion for real-time image-guided radiotherapy," Physics in Medicine and Biology, vol. 49, no. 3, pp. 425440, 2004.

[37] G. C. Sharp, S. B. Jiang, S. Shimizu, and H. Shirato, "Tracking errors in a prototype real-time tumour tracking system," Physics in Medicine and Biology, vol. 49, no. 23, pp. 5347-5356, 2004.

[38] J. W. Wong, M. B. Sharpe, D. A. Jaffray et al., "The use of active breathing control $(\mathrm{ABC})$ to reduce margin for breathing motion," International Journal of Radiation Oncology, Biology and Physics, vol. 44, no. 4, pp. 911-919, 1999.

[39] R. Garcia, R. Oozeer, H. L. Thanh et al., "Radiotherapy of lung cancer: the inspiration breath hold with a spirometric monitoring," Cancer/Radiotherapie, vol. 6, no. 1, pp. 30-38, 2002.

[40] K. E. Rosenzweig, J. Hanley, D. Mah et al., “The deep inspiration breath-hold technique in the treatment of inoperable non-small-cell lung cancer," International Journal of Radiation Oncology, Biology and Physics, vol. 48, no. 1, pp. 81-87, 2000.

[41] D. Mah, J. Hanley, K. E. Rosenzweig et al., "Technical aspects of the deep inspiration breath-hold technique in the treatment of thoracic cancer," International Journal of Radiation Oncology, Biology and Physics, vol. 48, no. 4, pp. 1175-1185, 2000.

[42] V. R. Kini, S. S. Vedam, P. J. Keall, S. Patil, C. Chen, and R. Mohan, "Patient training in respiratory-gated radiotherapy," Medical Dosimetry, vol. 28, no. 1, pp. 7-11, 2003.

[43] M. J. Murphy, “Tracking moving organs in real time," Seminars in Radiation Oncology, vol. 14, no. 1, pp. 91-100, 2004.

[44] P. H. Quanjer, G. J. Tammeling, J. E. Cotes, O. F. Pedersen, R. Peslin, and J. C. Yernault, "Lung volumes and forced ventilatory flows. Report working party standardization of lung function tests, european community for steel and coal. Official statement of the european respiratory society," The European Respiratory Journal Supplements, vol. 16, pp. 5-40, 1993.

[45] G. S. Mageras, E. Yorke, K. Rosenzweig et al., "Fluoroscopic evaluation of diaphragmatic motion reduction with a respiratory gated radiotherapy system," Journal of Applied Clinical Medical Physics, vol. 2, no. 4, pp. 191-200, 2001.
[46] L. Papiez, "The leaf sweep algorithm for an immobile and moving target as an optimal control problem in radiotherapy delivery," Mathematical and Computer Modelling, vol. 37, no. 78, pp. 735-745, 2003.

[47] B. Warkentin, P. Stavrev, N. Stavreva, C. Field, and B. G. Fallone, "A TCP-NTCP estimation module using DVHs and known radiobiological models and parameter sets," Journal of Applied Clinical Medical Physics, vol. 5, no. 1, pp. 50-63, 2004.

[48] S. D. McCarter and W. A. Beckham, "Evaluation of the validity of a convolution method for incorporating tumour movement and set-up variations into the radiotherapy treatment planning system," Physics in Medicine and Biology, vol. 45, no. 4, pp. 923931, 2000.

[49] I. J. Chetty, M. Rosu, D. L. McShan, B. A. Fraass, J. M. Balter, and R. K. Ten Haken, "Accounting for center-of-mass target motion using convolution methods in Monte Carlo-based dose calculations of the lung," Medical Physics, vol. 31, no. 4, pp. 925932, 2004.

[50] B. S. Yi, J. Perks, R. Houston, R. Stern, J. A. Purdy, and A. M. Chen, "Changes in position and volume of lung cancer target volumes during stereotactic body radiotherapy (SBRT): is image guidance necessary?" Technology in Cancer Research and Treatment, vol. 10, no. 5, pp. 495-504, 2011.

[51] L. Simon, P. Giraud, V. Servois, and J. C. Rosenwald, "Comparative study and clinical implementation of two breathingadapted radiotherapy techniques: dosimetric benefits for lung cancer treatment," Cancer/Radiotherapie, vol. 10, no. 6-7, pp. 370-376, 2006.

[52] E. Rietzel, T. Pan, and G. T. Y. Chen, "Four-dimensional computed tomography: image formation and clinical protocol," Medical Physics, vol. 32, no. 4, pp. 874-889, 2005.

[53] J. M. Balter, J. N. Wright, L. J. Newell et al., "Accuracy of a wireless localization system for radiotherapy," International Journal of Radiation Oncology, Biology and Physics, vol. 61, no. 3, pp. 933-937, 2005.

[54] R. I. Berbeco, S. B. Jiang, G. C. Sharp, G. T. Y. Chen, H. Mostafavi, and H. Shirato, "Integrated radiotherapy imaging system (IRIS): design considerations of tumour tracking with linac gantry-mounted diagnostic X-ray systems with flat-panel detectors," Physics in Medicine and Biology, vol. 49, no. 2, pp. 243-255, 2004.

[55] G. T. Y. Chen, J. H. Kung, and K. P. Beaudette, "Artifacts in computed tomography scanning of moving objects," Seminars in Radiation Oncology, vol. 14, no. 1, pp. 19-26, 2004.

[56] Q. S. Chen, M. S. Weinhous, F. C. Deibel, J. P. Ciezki, and R. M. Macklis, "Fluoroscopic study of tumor motion due to breathing: facilitating precise radiation therapy for lung cancer patients," Medical Physics, vol. 28, no. 9, pp. 1850-1856, 2001.

[57] J. Duan, S. Shen, J. B. Fiveash, I. A. Brezovich, R. A. Popple, and P. N. Pareek, "Dosimetric effect of respiration-gated beam on IMRT delivery," Medical Physics, vol. 30, no. 8, pp. 2241-2252, 2003.

[58] P. J. Keall, G. S. Mageras, J. M. Balter et al., “The management of respiratory motion in radiation oncology report of AAPM task group 76," Medical Physics, vol. 33, no. 10, pp. 3874-3900, 2006.

[59] S. S. Vedam, P. J. Keall, V. R. Kini, and R. Mohan, "Determining parameters for respiration-gated radiotherapy," Medical Physics, vol. 28, no. 10, pp. 2139-2146, 2001.

[60] L. Simon, P. Giraud, J. L. Dumas et al., "Practical recommendations for breathing-adapted radiotherapy," Cancer/Radiotherapie, vol. 11, no. 4, pp. 214-224, 2007. 
[61] C. R. Ramsey, D. D. Scaperoth, and D. C. Arwood, "Clinical experience with a commercial respiratory gating system," International Journal of Radiation Oncology, Biology and Physics, vol. 48, pp. 164-165, 2000.

[62] S. B. Jiang, "Technical aspects of image-guided respirationgated radiation therapy," Medical Dosimetry, vol. 31, no. 2, pp. 141-151, 2006.

[63] R. W. M. Underberg, F. J. Lagerwaard, B. J. Slotman, J. P. Cuijpers, and S. Senan, "Benefit of respiration-gated stereotactic radiotherapy for stage I lung cancer: an analysis of 4DCT datasets," International Journal of Radiation Oncology, Biology and Physics, vol. 62, no. 2, pp. 554-560, 2005.

[64] A. M. Berson, R. Emery, L. Rodriguez et al., "Clinical experience using respiratory gated radiation therapy: comparison of freebreathing and breath-hold techniques," International Journal of Radiation Oncology, Biology and Physics, vol. 60, no. 2, pp. 419426, 2004.

[65] K. E. Sixel, M. C. Aznar, and Y. C. Ung, "Deep inspiration breath hold to reduce irradiated heart volume in breast cancer patients," International Journal of Radiation Oncology, Biology and Physics, vol. 49, no. 1, pp. 199-204, 2001.

[66] H. M. Lu, E. Cash, M. H. Chen et al., "Reduction of cardiac volume in left-breast treatment fields by respiratory maneuvers: a CT study," International Journal of Radiation Oncology, Biology and Physics, vol. 47, no. 4, pp. 895-904, 2000.

[67] L. Simon, P. Giraud, V. Servois, and J. C. Rosenwald, "Lung volume assessment for a cross-comparison of two breathingadapted techniques in radiotherapy," International Journal of Radiation Oncology, Biology and Physics, vol. 63, no. 2, pp. 602609, 2005.

[68] H. D. Kubo and L. Wang, "Introduction of audio gating to further reduce organ motion in breathing synchronized radiotherapy," Medical Physics, vol. 29, no. 3, pp. 345-350, 2002.

[69] E. Yorke, K. E. Rosenzweig, R. Wagman, and G. S. Mageras, "Interfractional anatomic variation in patients treated with respiration-gated radiotherapy," Journal of Applied Clinical Medical Physics, vol. 6, no. 2, pp. 19-32, 2005.

[70] C. Ozhasoglu and M. J. Murphy, "Issues in respiratory motion compensation during external-beam radiotherapy," International Journal of Radiation Oncology, Biology and Physics, vol. 52, no. 5, pp. 1389-1399, 2002.

[71] G. S. Mageras and E. Yorke, "Deep inspiration breath hold and respiratory gating strategies for reducing organ motion in radiation treatment," Seminars in Radiation Oncology, vol. 14, no. 1, pp. 65-75, 2004.

[72] S. S. Vedam, V. R. Kini, P. J. Keall, V. Ramakrishnan, H. Mostafavi, and R. Mohan, "Quantifying the predictability of diaphragm motion during respiration with a noninvasive external marker," Medical Physics, vol. 30, no. 4, pp. 505-513, 2003.

[73] A. Schweikard, G. Glosser, M. Bodduluri, M. J. Murphy, and J. R. Adler, "Robotic motion compensation for respiratory movement during radiosurgery," Computer Aided Surgery, vol. 5, no. 4, pp. 263-277, 2000.

[74] H. Shirato, S. Shimizu, K. Kitamura et al., "Four-dimensional treatment planning and fluoroscopic real-time tumor tracking radiotherapy for moving tumor," International Journal of Radiation Oncology, Biology and Physics, vol. 48, no. 2, pp. 435-442, 2000.

[75] Y. Seppenwoolde, H. Shirato, K. Kitamura et al., "Precise and real-time measurement of 3D tumor motion in lung due to breathing and heartbeat, measured during radiotherapy,"
International Journal of Radiation Oncology, Biology and Physics, vol. 53, no. 4, pp. 822-834, 2002.

[76] S. Shimizu, H. Shirato, S. Ogura et al., "Detection of lung tumor movement in real-time tumor-tracking radiotherapy," International Journal of Radiation Oncology, Biology and Physics, vol. 51, no. 2, pp. 304-310, 2001.

[77] P. G. Seiler, H. Blattmann, S. Kirsch, R. K. Muench, and C. Schilling, "A novel tracking technique for the continuous precise measurement of tumour positions in conformal radiotherapy," Physics in Medicine and Biology, vol. 45, no. 9, pp. N103-N110, 2000.

[78] H. Shirato, S. Shimizu, T. Kunieda et al., "Physical aspects of a real-time tumor-tracking system for gated radiotherapy," International Journal of Radiation Oncology, Biology and Physics, vol. 48, no. 4, pp. 1187-1195, 2000.

[79] S. L. Meeks, J. M. Buatti, L. G. Bouchet et al., "Ultrasoundguided extracranial radiosurgery: technique and application," International Journal of Radiation Oncology, Biology and Physics, vol. 55, no. 4, pp. 1092-1101, 2003.

[80] R. I. Berbeco, H. Mostafavi, G. C. Sharp, and S. B. Jiang, "Towards fluoroscopic respiratory gating for lung tumours without radiopaque markers," Physics in Medicine and Biology, vol. 50, no. 19, pp. 4481-4490, 2005.

[81] W. D. D’Souza, S. A. Naqvi, and C. X. Yu, "Real-time intrafraction-motion tracking using the treatment couch: a feasibility study," Physics in Medicine and Biology, vol. 50, no. 17, pp. 4021-4033, 2005.

[82] M. J. Murphy, J. R. Adler, M. Bodduluri et al., "Image-guided radiosurgery for the spine and pancreas," Computer Aided Surgery, vol. 5, no. 4, pp. 278-288, 2000.

[83] S. Jiang, T. Bortfeld, A. Trofimov, E. Rietzel, G. Sharp, and N. Choi, "Synchronized moving aperture radiation therapy (SMART): treatment planning using 4D CT data," in Proceedings of the 14th International Conference on the Use of Computers in Radiation Therapy, Seoul, Korea, 2004.

[84] P. J. Keall, V. R. Kini, S. S. Vedam, and R. Mohan, "Motion adaptive $\mathrm{x}$-ray therapy: a feasibility study," Physics in Medicine and Biology, vol. 46, no. 1, pp. 1-10, 2001.

[85] T. Neicu, H. Shirato, Y. Seppenwoolde, and S. B. Jiang, "Synchronized moving aperture radiation therapy (SMART): average tumour trajectory for lung patients," Physics in Medicine and Biology, vol. 48, no. 5, pp. 587-598, 2003.

[86] P. J. Keall, S. Joshi, S. S. Vedam, J. V. Siebers, V. R. Kini, and R. Mohan, "Four-dimensional radiotherapy planning for DMLCbased respiratory motion tracking," Medical Physics, vol. 32, no. 4, pp. 942-951, 2005.

[87] L. Papiez, D. Rangaraj, and P. Keall, "Real-time DMLC IMRT delivery for mobile and deforming targets," Medical Physics, vol. 32, no. 9, pp. 3037-3048, 2005.

[88] L. Papiez and D. Rangaraj, "DMLC leaf-pair optimal control for mobile, deforming target," Medical Physics, vol. 32, no. 1, pp. 275-285, 2005.

[89] D. Rangaraj and L. Papiez, "Synchronized delivery of DMLC intensity modulated radiation therapy for stationary and moving targets," Medical Physics, vol. 32, no. 6, pp. 1802-1817, 2005.

[90] Y. Suh, B. Yi, S. Ahn et al., "Aperture maneuver with compelled breath (AMC) for moving tumors: a feasibility study with a moving phantom," Medical Physics, vol. 31, no. 4, pp. 760-766, 2004.

[91] K. K. Herfarth, J. Debus, F. Lohr et al., "Extracranial stereotactic radiation therapy: set-up accuracy of patients treated for liver 
metastases," International Journal of Radiation Oncology, Biology and Physics, vol. 46, no. 2, pp. 329-335, 2000.

[92] R. B. Case, D. J. Moseley, J. J. Sonke et al., "Interfraction and intrafraction changes in amplitude of breathing motion in stereotactic liver radiotherapy," International Journal of Radiation Oncology, Biology and Physics, vol. 77, no. 3, pp. 918-925, 2010.

[93] D. J. W. Kim, B. R. Murray, R. Halperin, and W. H. Y. Roa, "Held-breath self-gating technique for radiotherapy of nonsmall-cell lung cancer: a feasibility study," International Journal of Radiation Oncology, Biology and Physics, vol. 49, no. 1, pp. 4349, 2001.

[94] W. Wunderink, A. M. Romero, W. de Kruijf, H. de Boer, P. Levendag, and B. Heijmen, "Reduction of respiratory liver tumor motion by abdominal compression in stereotactic body frame, analyzed by tracking fiducial markers implanted in liver," International Journal of Radiation Oncology, Biology and Physics, vol. 71, no. 3, pp. 907-915, 2008.

[95] P. Giraud, E. Morvan, L. Claude et al., "Respiratory gating techniques for optimization of lung cancer radiotherapy," Journal of Thoracic Oncology, vol. 6, no. 12, pp. 2058-2068, 2011.

[96] P. Giraud, J. Djadi-Prat, M. Morelle et al., "Contribution of respiratory gating techniques for optimization of breast cancer radiotherapy," Cancer Investigation, vol. 30, no. 4, pp. 323-330, 2012.

[97] V. Beneyton, G. Billaud, C. Niederst et al., "Comparison of three dosimetric techniques to take in account lung tumor motion: gating-like technique results lead to advice the use of gating device even in the cases of pre-operative irradiation," Cancer/Radiotherapie, vol. 14, no. 1, pp. 50-58, 2010.

[98] P. Giraud, F. Reboul, S. Clippe et al., "Respiration-gated radiotherapy: current techniques and potential benefits," Cancer/Radiotherapie, vol. 7, supplement 1, pp. 15s-25s, 2003.

[99] R. Halperin, W. Roa, M. Field, J. Hanson, and B. Murray, "Setup reproducibility in radiation therapy for lung cancer: a comparison between T-bar and expanded foam immobilization devices," International Journal of Radiation Oncology, Biology and Physics, vol. 43, no. 1, pp. 211-216, 1999.

[100] H. D. Kubo, P. M. Len, S. I. Minohara, and H. Mostafavi, "Breathing-synchronized radiotherapy program at the University of California Davis Cancer Center," Medical Physics, vol. 27, no. 2, pp. 346-353, 2000.

[101] H. D. Kubo and B. C. Hill, "Respiration gated radiotherapy treatment: a technical study," Physics in Medicine and Biology, vol. 41, no. 1, pp. 83-91, 1996.

[102] F. J. Lagerwaard, J. R. van Sornsen de Koste, M. R. J. NijssenVisser et al., "Multiple "slow" CT scans for incorporating lung tumor mobility in radiotheraphy planning," International Journal of Radiation Oncology, Biology and Physics, vol. 51, no. 4, pp. 932-937, 2001.

[103] L. A. Dawson, K. K. Brock, S. Kazanjian et al., "The reproducibility of organ position using active breathing control (ABC) during liver radiotherapy," International Journal of Radiation Oncology, Biology and Physics, vol. 51, no. 5, pp. 1410-1421, 2001.

[104] W. A. Kalendar, R. Rienmuller, W. Seissler, J. Behr, M. Welke, and H. Fichte, "Measurement of pulmonary parenchymal attenuation: use of spirometric gating with quantitative CT," Radiology, vol. 175, no. 1, pp. 265-268, 1990.

[105] A. Pedersen, S. Korreman, H. Nyström, and L. Specht, "Efficacy of respiratory gated radiotherapy of breast cancer," Radiotherapy and Oncology, vol. 64, supplement 1, article S142, 2002.
[106] C. R. Ramsey, D. Scaperoth, and D. Arwood, "Clinical experience with a commercial respiratory gating system," International Journal of Radiation Oncology, Biology and Physics, vol. 48, pp. 164-165, 2000.

[107] V. M. Remouchamps, N. Letts, F. A. Vicini et al., "Initial clinical experience with moderate deep-inspiration breath hold using an active breathing control device in the treatment of patients with left-sided breast cancer using external beam radiation therapy," International Journal of Radiation Oncology, Biology and Physics, vol. 56, no. 3, pp. 704-715, 2003.

[108] S. Shen, J. Duan, J. B. Fiveash et al., "Validation of target volume and position in respiratory gated CT planning and treatment," Medical Physics, vol. 30, no. 12, pp. 3196-3205, 2003.

[109] C. D. Biancia, E. Yorke, C. S. Chui et al., "Comparison of end normal inspiration and expiration for gated intensity modulated radiation therapy (IMRT) of lung cancer," Radiotherapy and Oncology, vol. 75, no. 2, pp. 149-156, 2005.

[110] T. Zhang, H. Keller, M. J. O’Brien, T. R. Mackie, and B. Paliwal, "Application of the spirometer in respiratory gated radiotherapy," Medical Physics, vol. 30, no. 12, pp. 3165-3171, 2003.

[111] R. I. Berbeco, S. Nishioka, H. Shirato, G. T. Y. Chen, and S. B. Jiang, "Residual motion of lung tumours in gated radiotherapy with external respiratory surrogates," Physics in Medicine and Biology, vol. 50, no. 16, pp. 3655-3667, 2005.

[112] M. Morelle, R. Remonnay, P. Giraud, and M. O. Carrère, "Analyzing multiple learning effects in health care using multilevel modeling: application to radiotherapy at an early stage of innovation," International Journal of Technology Assessment in Health Care, vol. 25, no. 2, pp. 232-239, 2009.

[113] H. D. Kubo and L. Wang, "Compatibility of Varian 2100C gated operations with enhanced dynamic wedge and IMRT dose delivery," Medical Physics, vol. 27, no. 8, pp. 1732-1738, 2000.

[114] G. S. Mageras, E. Yorke, and S. B. Jiang, “4D’ IMRT delivery,” in Image-Guided IMRT, T. Bortfeld, R. Schmidt-Ullrich, W. de Neve, and D. E. Wazer, Eds., pp. 269-288, Springer, Heidelberg, Germany, 2005.

[115] E. Yorke, G. Mageras, T. LoSasso, H. Mostafavi, and C. Ling, "Respiratory gating of sliding window IMRT," in Proceedings of the 22nd Annual International Conference of the IEEE Engineering in Medicine and Biology Society, pp. 2118-2121, usa, July 2000.

[116] C. X. Yu, "Intensity-modulated arc therapy with dynamic multileaf collimation: an alternative to tomotherapy," Physics in Medicine and Biology, vol. 40, no. 9, pp. 1435-1449, 1995. 


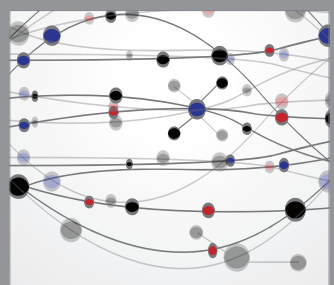

The Scientific World Journal
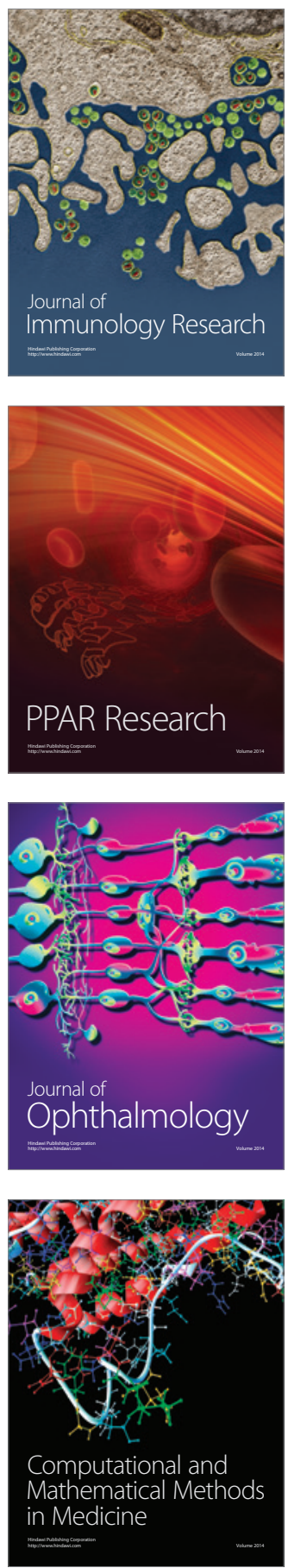

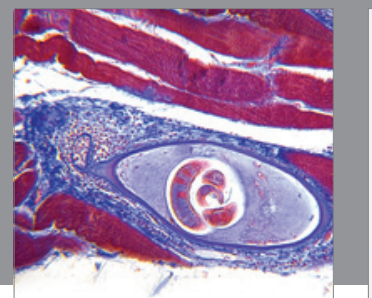

Gastroenterology

Research and Practice
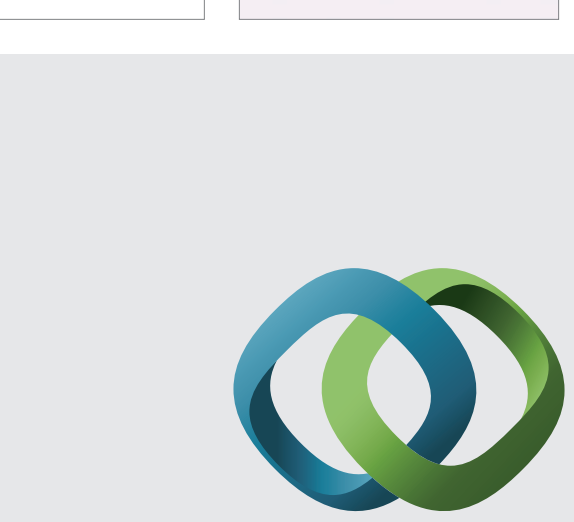

\section{Hindawi}

Submit your manuscripts at

http://www.hindawi.com
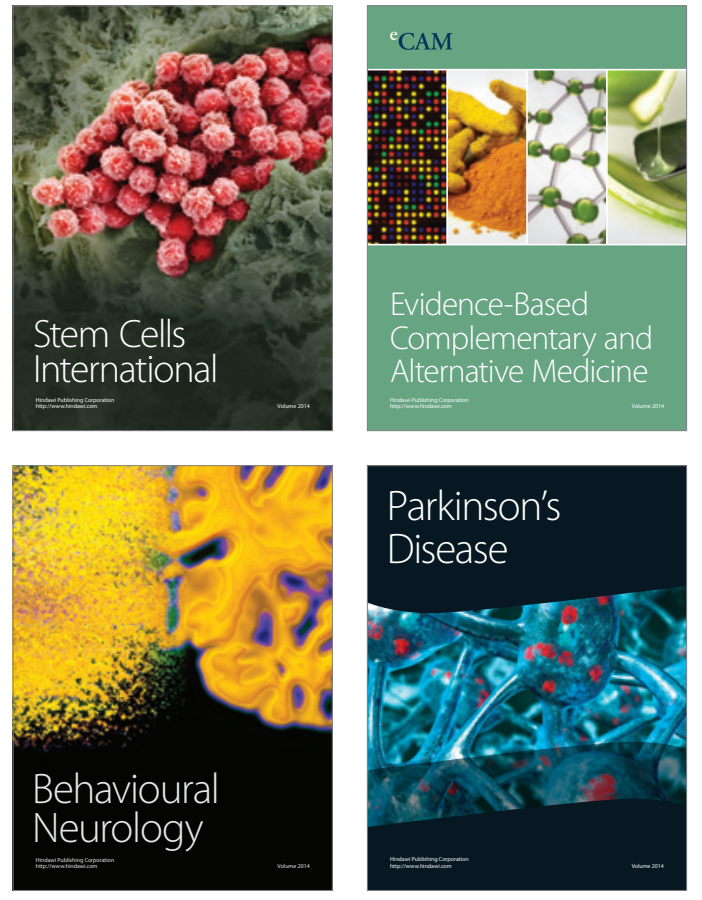
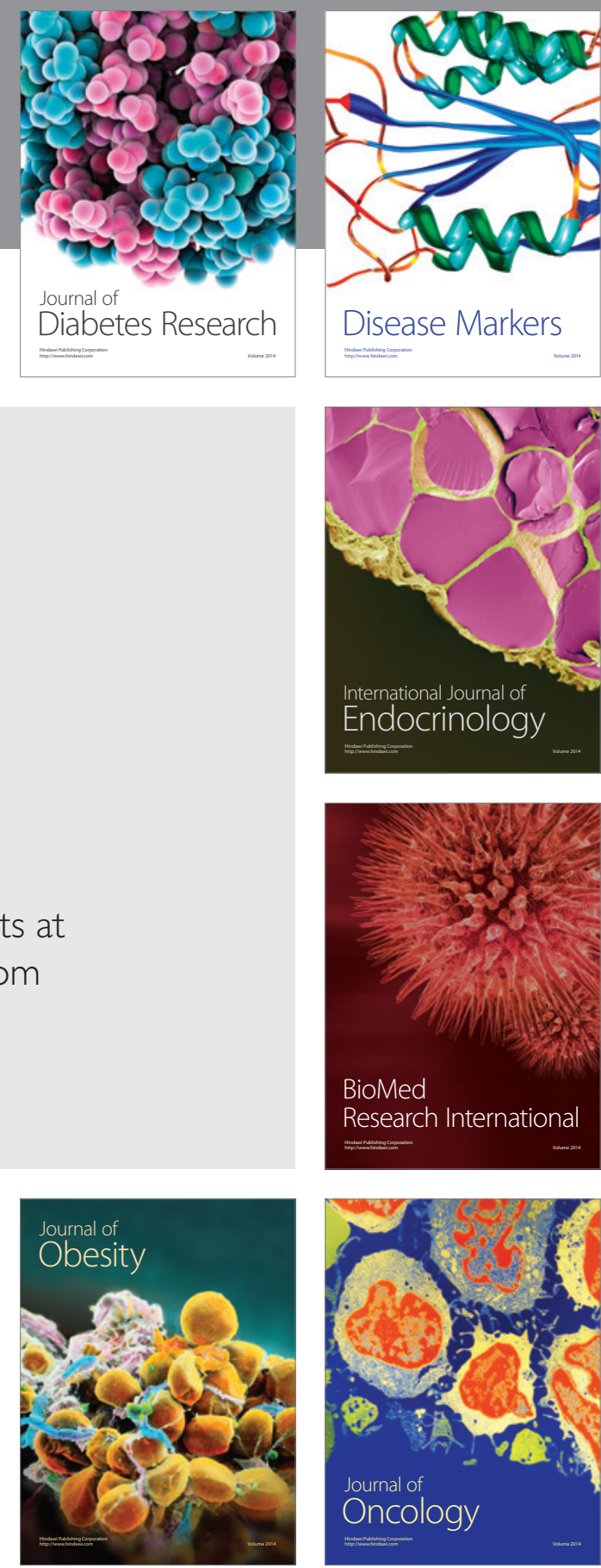

Disease Markers
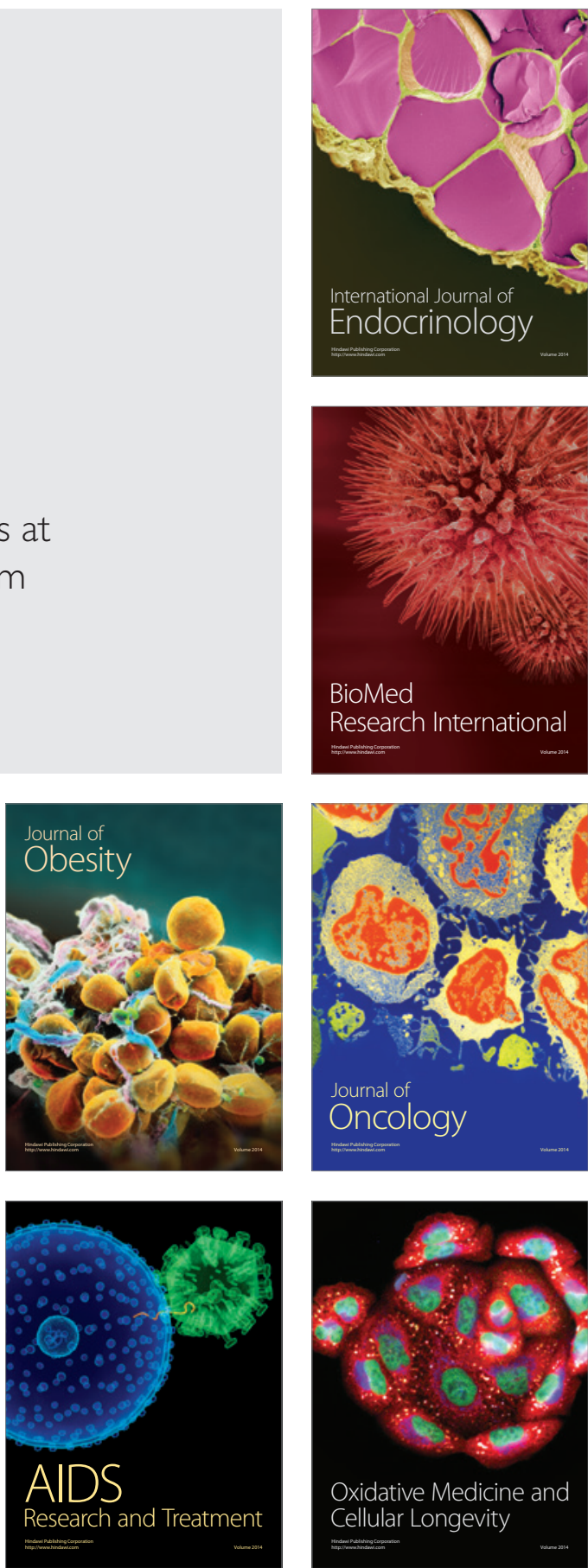